Draft Version OCTOBER 25, 2018

Preprint typeset using $\mathrm{L}^{A} \mathrm{~T}_{\mathrm{E}} \mathrm{X}$ style emulateapj v. 11/10/09

\title{
[O/Fe] ESTIMATES FOR CARBON-ENHANCED METAL-POOR STARS FROM NEAR-IR SPECTROSCOPY
}

Catherine R. Kennedy

Department of Physics \& Astronomy and JINA: Joint Institute for Nuclear Astrophysics, Michigan State University, East Lansing, MI 48824, USA

ThIRUPATHI SivarANi

Indian Institute of Astrophysics, 2nd Block, Koramangala, Bangalore 560034, India

Timothy C. Beers, Young Sun Lee

Department of Physics \& Astronomy and JINA: Joint Institute for Nuclear Astrophysics, Michigan State University, East Lansing, MI 48824, USA

Vinicius M. Placco, Silvia Rossi

Departamento de Astronomia - Instituto de Astronomia, Geofísica e Ciências Atmosféricas, Universidade de São Paulo, São Paulo, SP 05508-900, Brazil

Norbert Christlieb

Zentrum für Astronomie der Universität Heidelberg, Landessternwarte, Königstuhl 12, 69117, Heidelberg, Germany

FALK HERWIG

Department of Physics \& Astronomy, University of Victoria, Victoria, BC V8P5C2 Canada

Bertrand Plez

GRAAL-CNRS, Université Montpellier II - UMR 5024, Place Eugène Bataillon, F-34095, Montpellier Cedex 05, France (Accepted for publication in AJ - January 9, 2011)
Draft version October 25, 2018

\begin{abstract}
We report on oxygen abundances determined from medium-resolution near-IR spectroscopy for a sample of 57 carbon-enhanced metal-poor (CEMP) stars selected from the Hamburg/ESO survey. The majority of our program stars exhibit oxygen-to-iron ratios in the range $+0.5<[\mathrm{O} / \mathrm{Fe}]<+2.0$. The $[\mathrm{O} / \mathrm{Fe}]$ values for this sample are statistically compared to available high-resolution estimates for known CEMP stars, as well as to high-resolution estimates for a set of carbon-normal metal-poor stars. Carbon, nitrogen, and oxygen abundance patterns for a sub-sample of these stars are compared to yield predictions for very metal-poor asymptotic giant-branch abundances in the recent literature. We find that the majority of our sample exhibit patterns that are consistent with previously studied CEMP stars having s-process-element enhancements, and thus have very likely been polluted by carbon- and oxygen-enhanced material transferred from a metal-poor asymptotic giant-branch companion.

Subject headings: Galaxy: halo - stars: abundances - stars: Population II - stars: AGB - techniques: spectroscopic
\end{abstract}

\section{INTRODUCTION}

Carbon-enhanced metal-poor (CEMP) stars are quite common in the halo populations of the Milky Way, and are of particular interest, as they preserve important astrophysical information concerning the early chemical evolution of the Galaxy (Beers \& Christlieb 2005). Previous work has indicated that at least $20 \%$ of stars with metallicities $[\mathrm{Fe} / \mathrm{H}]<-2.0$ exhibit large over-

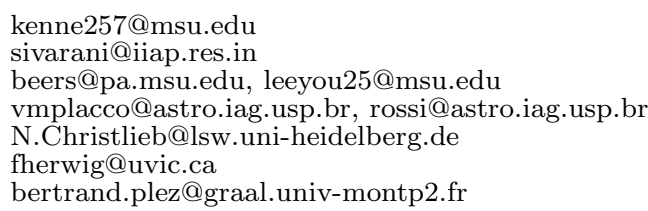

abundances of carbon $([\mathrm{C} / \mathrm{Fe}] i+1.0$; Lucatello et al. 2006; Marsteller et al. 2009), although recent studies (e.g. Cohen et al. 2005; Frebel et al. 2006), have claimed that this fraction is somewhat lower $(9 \%$ and $14 \%$, respectively, for $[\mathrm{Fe} / \mathrm{H}]<-2.0)$. In any case, the fraction of CEMP stars rises to $30 \%$ for $[\mathrm{Fe} / \mathrm{H}]<-3.0,40 \%$ for $[\mathrm{Fe} / \mathrm{H}]<-3.5$, and $100 \%$ for $[\mathrm{Fe} / \mathrm{H}]<-4.0$ (Beers \& Christlieb 2005; Frebel et al. 2005; Norris et al. 2007).

There exist a number of classes of CEMP stars, some of which have been associated with proposed progenitor objects. CEMP-s stars (those with s-process-element enhancement), for example, are the most commonly observed type to date. High-resolution spectroscopic studies have revealed that around $80 \%$ of CEMP stars exhibit 
s-process-element enhancement (Aoki et al. 2007). The favored mechanism invoked to account for these stars is mass transfer of carbon-enhanced material from the envelope of an asymptotic giant-branch (AGB) star to its binary companion; it is this surviving binary companion that is now observed as a CEMP-s star.

The class of CEMP-no stars (which exhibit no strong neutron-capture-element enhancements) are particularly prevalent among the most metal-poor stars. Possible progenitors for this class include massive, rapidlyrotating, mega metal-poor $([\mathrm{Fe} / \mathrm{H}]<-6.0)$ stars, which models suggest have greatly enhanced abundances of CNO due to distinctive internal burning and mixing episodes, followed by strong mass loss (Meynet et al. 2006; Hirschi et al. 2006; Meynet et al. 2010). Another suggested mechansim is pollution of the interstellar medium by so-called faint supernovae associated with the first generations of stars, which experience extensive mixing and fallback during their explosions (Umeda \& Nomoto 2003, 2005; Tominaga et al. 2007); high $[\mathrm{C} / \mathrm{Fe}]$ and $[\mathrm{O} / \mathrm{Fe}]$ ratios are predicted in the ejected material. This model well reproduces the observed abundance pattern of the CEMP-no star BD+44:493, the ninth-magnitude $[\mathrm{Fe} / \mathrm{H}]=-3.7$ star $($ with $[\mathrm{C} / \mathrm{Fe}]=+1.3$, $[\mathrm{N} / \mathrm{Fe}]=+0.3,[\mathrm{O} / \mathrm{Fe}]=+1.6)$ discussed by Ito et al. (2009).

The great majority of known CEMP stars were originally identified as metal-poor candidates from objectiveprism surveys, such as the HK survey (Beers et al. 1985, 1992), and the Hamburg/ESO Survey (HES; Christlieb 2003; Christlieb et al. 2008), based on a weak (or absent) Ca II K line. Some candidate CEMP stars also come from a list of HES stars selected from the prism plates based on their strong molecular lines of carbon (Christlieb et al. 2001). Medium-resolution spectra for most of these objects have been obtained over the past few years (Goswami et al. 2006; Marsteller 2007; Goswami et al. 2010, Sivarani et al., in preparation). Inspection of these data indicate that at least $50 \%$ of these targets are consistent with identification as CEMP stars, while the others are roughly solar-metallicity carbon-rich stars. Dedicated surveys for CEMP stars covering a wide range of carbon abundance and metallicities are just now getting underway, based on the observed strength of the CH G band measured from the HES prism plates (e.g., Placco et al. 2010).

In order to more fully test the association of CEMP-no stars with massive primordial stars and/or faint supernovae, and to better explore the nature of the s-process in low-metallicity AGB stars (which is still rather poorly understood; Herwig 2005), we require measurements of the important elements $\mathrm{C}, \mathrm{N}$, and $\mathrm{O}$ for as large a sample of CEMP stars as possible. While estimates of carbon and nitrogen abundances can be determined from medium-resolution optical or near-UV spectra of CEMP stars (e.g., Rossi et al. 2005; Beers et al. 2007b; Johnson et al. 2007; Marsteller et al. 2009), high-resolution spectroscopy is usually required in order to obtain estimates of oxygen abundances from the forbidden $[\mathrm{O} \mathrm{I}]$ $\lambda 6300 \AA$ line, the $\lambda 7700 \AA$ triplet (e.g., Schuler et al. 2006; Sivarani et al. 2006; Fabbian et al. 2009, and references therein), or the $\mathrm{OH}$ lines at 1.5-1.7 $\mu \mathrm{m}$ (Melendez \& Barbuy 2002). Masseron et al. (2010) provides a useful compilation of known elemental abundances for CEMP stars. In addition to abundance measurements for metal-poor halo stars, oxygen abundances have also been measured directly in the gas phase in damped Lyman $\alpha$ systems (Pettini et al. 2002, 2008).

If a star has a measured carbon abundance (and, assuming $\mathrm{C} / \mathrm{O}>1$, which applies for most CEMP stars), essentially all of the $\mathrm{O}$ is locked up in $\mathrm{CO}$ molecules, and medium-resolution spectroscopy of the $\mathrm{CO}$ ro-vibrational bands in the near-IR can be used for estimation of [O/Fe] (e.g., Beers et al. 2007b, and references therein). Although one sacrifices measurement accuracy, relative to high-resolution studies, this approach has the great advantage that medium-resolution spectroscopy can be gathered far faster than high-resolution spectroscopy, ensuring that much larger samples of stars can be investigated. In addition, the large separation of the ${ }^{13} \mathrm{CO}$ lines from the ${ }^{12} \mathrm{CO}$ lines at $2.3 \mu \mathrm{m}$ provides a straightforward means to measure the important mixing diagnostic ${ }^{12} \mathrm{C} /{ }^{13} \mathrm{C}$, as long as the $\mathrm{S} / \mathrm{N}$ of the spectra are sufficient.

This paper is outlined as follows. In Section 2 we discuss details of the observations and data reduction procedures used in the present study. Section 3 describes the previously determined atmospheric parameter estimates and their origins, as well as details about the synthetic spectra. Methods used for determination of $[\mathrm{O} / \mathrm{Fe}]$ for our sample of stars are described in Section 4. Our results, and a statistical comparison to high-resolution estimates of $[\mathrm{C} / \mathrm{Fe}]$ and $[\mathrm{O} / \mathrm{Fe}]$ for a subset of our program stars can be found in Section 5 . Section 6 is a short discussion of our results; conclusions follow in Section 7.

\section{OBSERVATIONS AND DATA REDUCTION}

Our sample of 57 stars was selected from the Hamburg/ESO Survey (HES), based on follow-up mediumresolution optical spectra obtained during the course of searches for low-metallicity stars. These optical spectra were obtained with the GOLDCAM spectrograph on the KPNO 2.1m telescope and with the RC Spectrographs on the $4 \mathrm{~m} \mathrm{KPNO}$ and CTIO telescopes (see Beers et al. 2007b, hereafter Paper I). Additional targets were selected from the list of carbon-rich candidates published by Christlieb et al. (2001) with available optical spectra. Based on the optical spectra, all of the candidates are metal-poor stars, spanning the metallicity range $-2.8 \leq[\mathrm{Fe} / \mathrm{H}] \leq-1.0$. All of the stars were selected to be carbon-rich, with the majority exhibiting $[\mathrm{C} / \mathrm{Fe}] \geq+1.0$, and thus are carbon-enhanced as defined by Beers \& Christlieb (2005). Since our intention was to obtain near-IR spectroscopy of the CO features, the stars were also selected to have effective temperatures less than $5000 \mathrm{~K}$, since warmer stars do not exhibit strong CO.

Estimates of $[\mathrm{O} / \mathrm{Fe}]$ for our program stars are derived from analysis of medium-resolution near-IR spectra taken with the SOAR $4.1 \mathrm{~m}$ telescope, using the OSIRIS (Ohio State InfraRed Imager/Spectrometer; Depoy et al. 1993) spectrograph during October 2005 to June 2008. We used the long slit (width set to $1^{\prime \prime}$ ) and long camera (with focal ratio $\mathrm{f} / 7$ ), which provided a resolving power $R=3000$. The long-pass K-band filter was used to isolate the spectral region from $2.25 \mu \mathrm{m}$ to $2.45 \mu \mathrm{m}$. Visible in this band are the four ro-vibrational CO features used for the determination of $[\mathrm{O} / \mathrm{Fe}]$. We also observed A0type stars at the same airmass as the observations of 
the program objects in order to correct for the presence of telluric lines in the spectra. The $K$-band magnitude range for our sample stars is $\sim 7-12$, resulting in exposure times in the range $600-2400$ seconds in order to reach our targeted $\mathrm{S} / \mathrm{N}$ ratio of 50/1. Spectra of ArNe arc lamps, taken before or after each program star, were used for the wavelength calibration of our sample. Bias correction, flat-fielding, spectral extraction, wavelength calibration, telluric feature correction, and continuum normalization were all performed using standard IRAF packages 1 .

\section{ADOPTED ATMOSPHERIC PARAMETERS AND SYNTHETIC SPECTRA}

Atmospheric parameters ( $T_{\text {eff }}, \log g$, and $\left.[\mathrm{Fe} / \mathrm{H}]\right)$ were estimated from available optical and near-IR photometry, as well as from previously obtained mediumresolution optical spectroscopy. Estimates of $T_{\text {eff }}$ are obtained from measured $\mathrm{V}-\mathrm{K}$ colors (taken from Beers et al. 2007a, and references therein, as well as from the 2MASS Point Source Catalog; Skrutskie et al. 2006). The use of near-IR photometry provides for a more accurate determination of $T_{\text {eff }}$, as the $\mathrm{K}$ band is less influenced by the presence of carbon features than bluer bands. We used the Alonso et al. (1996) calibrations of $T_{\text {eff }}$ with $\mathrm{V}-\mathrm{K}$ colors, as described in Paper I. Surface gravities, $\log g$, have been been estimated based on the Padova evolutionary tracks for metallicities $[\mathrm{Fe} / \mathrm{H}]=-2.5$ and $[\mathrm{Fe} / \mathrm{H}]=-1.7$ (Girardi et al. 2000; Marigo et al. 2001). Uncertainties in $T_{\text {eff }}$ and $\log g$ are $100 \mathrm{~K}$ and 0.5 dex, respectively. The microturbulence is taken to be $2 \mathrm{~km}$ $\mathrm{s}^{-1}$ for all stars. This is consistent with previouslydetermined microturbulence values for giant CEMP stars (Johnson et al. 2007; Aoki et al. 2007).

We have constructed two sets of synthetic spectral templates, covering the optical and near-IR bands. Each set consists of 2000 synthetic spectra with carbon-enhanced atmospheres generated with the MARCS code (Gustafsson et al. 2008). We used a previous generation of models here, as updated CEMP models were not available. We do not, however, anticipate large differences in the models of spectra. The use of carbon-enhanced models is of particular importance for cool CEMP stars, for which the atmospheric structure is significantly altered by carbon (Masseron et al. 2006). No 3D $\rightarrow 1 \mathrm{D}$ corrections have been applied to our estimates. Recent studies of these effects on two hyper metal-poor stars (Collet et al. 2006) have revealed $[\mathrm{O} / \mathrm{Fe}]$ corrections of $\sim-0.8$ based on $\mathrm{OH}$ molecules, thereby lowering the measured abundance of oxygen. However, the magnitude of such corrections is expected to decrease with increasing metallicity (Collet et al. 2007). As the metallicities of our targets range from -1.0 to -2.8 , it is likely that the $3 \mathrm{D}$ effects are less severe. In addition, more recent studies have been carried out (A. Ivanauskas, private communication) concerning the effects of convection on $\mathrm{C}_{2}, \mathrm{CH}, \mathrm{CN}, \mathrm{CO}, \mathrm{NH}$, and $\mathrm{OH}$ molecules. When compared to the results from Collet et al. (2006), the magnitude of the corrections appear smaller.

1 IRAF is distributed by the National Optical Astronomy Observatories, which is operated by the Association of Universities for Research in Astronomy, Inc. under cooperative agreement with the National Science Foundation.
The synthetic grid covers a range $T_{\text {eff }}=4000$ to $6000 \mathrm{~K}$ , $\log g=0.0$ to $5.0,[\mathrm{Fe} / \mathrm{H}]=-5.0$ to 0.0 , and $[\mathrm{C} / \mathrm{Fe}]$ $=0.0$ to +4.0 . We adopt fixed nitrogen abundances set to 0.5 dex less than the carbon abundances, which is roughly appropriate for CEMP stars. The $\mathrm{CH}$ and $\mathrm{CN}$ linelists used for the synthesis of the optical spectra are those compiled by Plez (see Plez \& Cohen 2005). The $\mathrm{CO}$ linelists used for the near-IR synthesis are taken from Kurucz (1993). The synthetic grids are then degraded to match the resolving power of the observed spectra ( $R=2000$ for the optical spectra, $R=3000$ for the near-IR spectra).

The optical spectra are used for the determination of $[\mathrm{Fe} / \mathrm{H}]$ and $[\mathrm{C} / \mathrm{H}]$. The $\mathrm{Ca}$ II $\mathrm{K}$ line is matched with the model spectra to estimate $[\mathrm{Fe} / \mathrm{H}]$, and the $\mathrm{C}_{2}$ and $\mathrm{CN}$ features are fit for the estimation of $[\mathrm{C} / \mathrm{H}]$ (see Paper I). Our adopted atmospheric parameters, as well as the derived $[\mathrm{C} / \mathrm{H}]$ and $[\mathrm{C} / \mathrm{Fe}]$, are listed in columns (2)-(6) of Table 1 .

\section{DETERMINATION OF $[\mathrm{O} / \mathrm{H}]$}

In order to determine $[\mathrm{O} / \mathrm{H}]$ we employed the near-IR synthetic spectra constructed from model atmospheres with carbon enhancements (see above). Each synthetic spectrum covers the wavelength range $2.25 \mu \mathrm{m}-2.40 \mu \mathrm{m}$. For all combinations of these parameters, models were available with $[\mathrm{O} / \mathrm{Fe}]$ values of $0.0,+0.4$, and +0.8 .

The first step in the determination of $[\mathrm{O} / \mathrm{Fe}]$ is to use the grid of synthetic spectra in combination with the atmospheric parameters to create three models with $[\mathrm{O} / \mathrm{Fe}]$ values of $0.0,+0.4$, and +0.8 . These models are then used in order to estimate the $[\mathrm{O} / \mathrm{Fe}]$ of the program stars. Each star in the sample has a previously estimated $T_{\text {eff }}$, $\log g,[\mathrm{Fe} / \mathrm{H}]$, and $[\mathrm{C} / \mathrm{Fe}]$. Given the fact that four parameters are known, the routine begins with 48 models: 16 models for each value of $[\mathrm{O} / \mathrm{Fe}]$. The 48 models are selected as having the two closest values of $T_{\text {eff }}, \log g$, $[\mathrm{Fe} / \mathrm{H}]$, and $[\mathrm{C} / \mathrm{Fe}]$ for each of the three values of $[\mathrm{O} / \mathrm{Fe}]$. Once selected, a linear interpolation over each parameter is performed in order to create three final models, one for each value of $[\mathrm{O} / \mathrm{Fe}]$, with the known values of the four parameters. The three final models for a set of typical atmospheric parameters are shown in Figure 1. With the other parameters fixed, it is easy to see how a typical spectrum changes with increasing oxygen abundance. Next, a linear interpolation over $[\mathrm{O} / \mathrm{Fe}]$ is performed on the three final models, creating new model spectra with varying oxygen. It should be noted that while the interpolation was performed over $[\mathrm{O} / \mathrm{Fe}]$, the metallicity is fixed, and therefore the oxygen varies as $[\mathrm{O} / \mathrm{H}]$. In some cases, it was necessary to extrapolate beyond the boundaries of the model grid in order to find a good fit to the data.

Due to the presence of four large CO band in the nearIR spectra, there were difficulties in fitting the continuum across the entire region. It was important to fit the continua with a low-order function so that the depth of the absorption features were not artificially enhanced or lessened due to the continuum fit. For this reason, the spectra of all stars in the sample were trimmed around each of four CO bands, and a local continuum was fit for each band prior to spectral synthesis. With the use of the synthetic spectra, oxygen abundances were then estimated individually for each of the four bands by min- 
imizing $\chi^{2}$. A robust average using bisquare weighting of the four separate values was taken as the final estimate of oxygen abundance, with an associated robust estimate of the scatter in these values taken as the error of determination.

Figure 2 shows the fitting technique applied to four stars from the sample. Each row shows the four separate estimates of $[\mathrm{O} / \mathrm{H}]$ for each star. Also plotted on each panel are synthetic spectra with $[\mathrm{O} / \mathrm{H}]$ values that vary from the best-fitting spectra by \pm 0.5 dex. Once the robust average is applied the resulting estimates of $[\mathrm{O} / \mathrm{H}]$ are -1.2 for HE $0111-1346,-0.5$ for HE 0519-2053, -0.6 for HE $1207-3156$, and -1.2 for HE 2339-0837.

\section{RESULTS}

The distributions of $[\mathrm{O} / \mathrm{Fe}]$ versus three of the parameters used for their determinations are shown in Figure 3. The solid lines are linear fits to the data. For the entire sample, there are no significant correlations of $[\mathrm{O} / \mathrm{Fe}]$ with $T_{\text {eff }},[\mathrm{Fe} / \mathrm{H}]$, or $[\mathrm{C} / \mathrm{Fe}]$. In the middle panel of Figure 3 , it can be seen that only one of the stars in our sample with $[\mathrm{Fe} / \mathrm{H}]<-2.5$ has a value of $[\mathrm{O} / \mathrm{Fe}]$ less than +1.0 . The bottom panel of Figure 3 shows the distribution of $[\mathrm{O} / \mathrm{Fe}]$ versus $[\mathrm{C} / \mathrm{Fe}]$, revealing that the majority of our sample (45 stars) have $[\mathrm{C} / \mathrm{Fe}]>+1.0$, and so meet the definition for CEMP stars given by Beers \& Christlieb (2005). The other stars exhibit carbon enhancements of $[\mathrm{C} / \mathrm{Fe}] \geq+0.5$, and thus are at least moderately enhanced in carbon. The average error in the determination of $[\mathrm{O} / \mathrm{Fe}]$ for our entire sample is 0.4 dex. We adopted a minimum error for our $[\mathrm{O} / \mathrm{Fe}]$ estimates of 0.25 dex due to the influence of errors that arise from the estimation of $T_{\text {eff }}, \log g,[\mathrm{Fe} / \mathrm{H}]$, and $[\mathrm{C} / \mathrm{Fe}]$ (see Paper I for details).

In Figure 4, a carbon cut has been made, such that only the oxygen abundances for those stars with $[\mathrm{C} / \mathrm{Fe}]$ $>+1.75$ are plotted against $[\mathrm{Fe} / \mathrm{H}]$. The stars with the highest abundances of carbon exhibit some of the lowest metallicities in our sample. This is not surprising, given that high values of $[\mathrm{C} / \mathrm{Fe}]$ are often associated with lower metallicities. The fit to the data shows a slight trend of increasing oxygen with decreasing metallicity. The solid line is a least squares fit of $[\mathrm{O} / \mathrm{Fe}]$ as a function of $[\mathrm{Fe} / \mathrm{H}]$. Only a marginally significant slope $(-0.616 \pm 0.314)$ is found, hence the correlation is quite weak. For comparison, the dashed line in this figure represents the the fit of $[\mathrm{O} / \mathrm{Fe}]$ versus $[\mathrm{Fe} / \mathrm{H}]$ for the carbon-normal stars from the Spite et al. (2005) sample. The $[\mathrm{O} / \mathrm{Fe}]$ estimates of the Spite et al. (2005) sample come from the forbidden [O I] $\lambda 6300 \AA$ line.

\subsection{Statistical Comparison to High-Resolution Oxygen Estimates}

The present catalog of measured oxygen abundances available in the literature for CEMP stars is still relatively small, due to the difficulty of obtaining estimates from optical spectra, even at high spectral resolution. However, we can at least compare the regions of the $[\mathrm{O} / \mathrm{Fe}]$ vs. $[\mathrm{Fe} / \mathrm{H}]$ parameter space that are occupied by CEMP stars of various sub-classes, based on previous high-resolution oxygen estimates, with those from our present medium-resolution effort.

Figure 5 shows $[\mathrm{O} / \mathrm{Fe}]$ for our entire sample, with different boxes indicating the regions of parameter space occupied by several classes of CEMP stars. Sources for the high-resolution data for different classes of CEMP stars can be found in Masseron et al. (2010), and references therein. The majority of the stars in our sample occupy regions of the diagram as CEMP stars that have confirmed, high-resolution measurements of s-processelement enhancements. There is clearly overlap with the region occupied by CEMP-r/s stars as well. Few of our stars overlap with the region occupied by CEMP-no stars in the literature; CEMP-no stars tend to be more metaldeficient than most of the stars in our sample.

\subsection{High-Resolution Nitrogen Estimates}

For 13 of our program stars, high-resolution estimates of $[\mathrm{N} / \mathrm{Fe}]$ are available from S. Lucatello (private communication) and/or Aoki et al. (2007). We selected ten of these stars for which the available highresolution estimates of $[\mathrm{C} / \mathrm{Fe}]$ were within 0.5 dex of our medium-resolution estimates. The three that are omitted from our analysis and discussion have associated high-resolution $[\mathrm{Fe} / \mathrm{H}]$ and/or $[\mathrm{C} / \mathrm{H}]$ estimates that differ significantly from the medium-resolution estimates of these species. We report values of high-resolution $[\mathrm{C} / \mathrm{Fe}]$, $[\mathrm{C} / \mathrm{Fe}]_{h}$, using our estimates of $[\mathrm{Fe} / \mathrm{H}]$ combined with the high-resolution $[\mathrm{C} / \mathrm{H}]$. We report high-resolution estimates of $[\mathrm{N} / \mathrm{Fe}],[\mathrm{N} / \mathrm{Fe}]_{h}$, by combining our estimates of $[\mathrm{Fe} / \mathrm{H}]$ with the high-resolution values of $[\mathrm{N} / \mathrm{H}]$. Two of the ten stars had high-resolution estimates both from Lucatello and Aoki et al. (2007), and an average of the two available estimates was taken.

The values of $T_{\text {eff }}, \log g,[\mathrm{Fe} / \mathrm{H}],[\mathrm{C} / \mathrm{H}],[\mathrm{C} / \mathrm{Fe}]$, $[\mathrm{C} / \mathrm{Fe}]_{h},[\mathrm{~N} / \mathrm{Fe}]_{h},[\mathrm{O} / \mathrm{H}],[\mathrm{O} / \mathrm{Fe}]$, and $\sigma_{[O / F e]}$ for our entire sample are listed in Table 1.

\section{DISCUSSION}

We expect that the majority of CEMP stars in our sample have been polluted by a companion lowmetallicity AGB star. In an AGB star, intershell oxygen is predicted to be closely related to intershell ${ }^{12} \mathrm{C}$, which in turn has a direct influence on the maximum ${ }^{13} \mathrm{C}$ abundance. By studying these abundances, we can better understand the nature of the s-process, as the ${ }^{13} \mathrm{C}(\alpha, n){ }^{16} \mathrm{O}$ reaction is a major neutron source for the s-process in AGB stars (Lugaro et al. 2003). According to current theory, oxygen production in AGB stars becomes increasingly significant with decreasing metallicity (Herwig 2004, 2005; Campbell \& Lattanzio 2008; Lau et al. 2009). Since the overabundance of oxygen is smaller at solar metallicities, lower metallicities are better suited for probing the primary production of oxygen. In addition, the observed abundance patterns of elements produced by the progenitor is expected to depend on the mass (and metallicity) of the AGB star (Herwig 2004; Stancliffe \& Glebbeek 2008).

In the following subsections we consider a number of issues that could potentially impact the interpretation of our measurements. First, we compare the results of our CEMP stars to those of carbon-normal metal-poor stars (Section 6.1). We then turn to recent literature on the abundance yields of low-metallicity AGB models to compare with our derived $\mathrm{C}, \mathrm{N}$, and $\mathrm{O}$ abundances (Section 6.2). We also consider how dilution processes might be expected to alter our abundances (Section 6.3). Finally, we address the sources of uncertainty in the AGB 
models, and how these may lead to altered abundances of $\mathrm{C}, \mathrm{N}$, and $\mathrm{O}$ (Section 6.4).

\section{1. $[\mathrm{O} / \mathrm{Fe}]$ in Carbon-Normal and Carbon-Enhanced Metal-Poor Stars}

The linear fit to the full sample shown in the middle panel of Figure 3 is consistent with the fit from the Spite et al. (2005) sample. However, most of the values of $[\mathrm{O} / \mathrm{Fe}]$ from the carbon-normal sample are tightly distributed around a constant value of +0.7 . Referring back to Figure 5, we see much more scatter in our values of $[\mathrm{O} / \mathrm{Fe}]$ for the carbon-enhanced stars, with values reaching as high as +2.0 . It can be inferred that metalpoor stars, regardless of carbon-enhancement, commonly exhibit enhancements of oxygen. However, when carbon enhancement is present, additional oxygen enhancement can be expected as well, due to the fact that both of these elements are enhanced by some of the same mechanisms.

\section{2. $C, N$, and $O$ : Comparison with AGB Models}

For 10 of the 13 stars for which we report highresolution $[\mathrm{N} / \mathrm{Fe}]$ estimates in Table 1, Figure 6 shows $[\mathrm{C} / \mathrm{Fe}],[\mathrm{N} / \mathrm{Fe}]$, and $[\mathrm{O} / \mathrm{Fe}]$ as a function of $[\mathrm{Fe} / \mathrm{H}]$. One can notice an increase in the abundances of all three species with decreasing metallicity. The linear fits for each of the species all have approximately the same slope, suggesting that abundances of carbon, nitrogen, and oxygen are highly correlated with one another. With estimates of $[\mathrm{C} / \mathrm{Fe}],[\mathrm{N} / \mathrm{Fe}]$, and $[\mathrm{O} / \mathrm{Fe}]$, we can compare our results to the predictions of abundance yields due to AGB evolution as described by Herwig (2004). Both carbon and oxygen are dredged up to the surface in AGB stars after thermal pulses. The overabundance of these elements in low-metallicity AGB stars is larger for lower initial masses (Herwig 2004), due to the fact that the intershell mass is larger. With a similarly large dredge up parameter and a smaller envelope, the enrichment of $\mathrm{C}$ and $\mathrm{O}$ in the envelope of a lower mass star is larger. This can be seen in the top panel of Figure 7, where the abundance predictions for C, N, and O (Herwig 2004) are shown for five different AGB masses, ranging from $2 \mathrm{M}_{\odot}$ to $6 \mathrm{M}_{\odot}$, all with $[\mathrm{Fe} / \mathrm{H}]=-2.3$. The $\mathrm{C}$, $\mathrm{N}$, and $\mathrm{O}$ abundances for ten stars with available $[\mathrm{N} / \mathrm{Fe}]$ in our sample are shown in a similar way in the lower two panels of Figure 7. For most of the stars, the relationship of carbon and oxygen is consistent with the models. The abundance pattern for HE $0017+0055$ is a very close match to an AGB star of about $3 \mathrm{M}_{\odot}$. The other nine stars exhibit some discrepancy with respect to nitrogen, which has been noted before for other CEMP stars with s-process element enhancement (Paper I; Sivarani et al. 2006). A previous effort to search for metal-poor stars with large enhancements of nitrogen relative to carbon yielded similar abundances (Johnson et al. 2007). All but five of the stars in our sample are giants, and thus more mixing and dilution of any material transferred from an AGB companion is expected, thereby resulting in such intermediate abundances of nitrogen (Paper I; Denissenkov \& Pinsonneault 2008). In addition, the possible occurence of H-ingestion flashes (HIF; Herwig 2003, 2005; Woodward et al. 2008; Hajduk et al. 2005; Campbell \& Lattanzio 2008) could potentially enhance $\mathrm{N}$ in metal-poor stars.
Nitrogen is an element that is very sensitive to $\mathrm{CN}$ cycling, and the $\mathrm{C} / \mathrm{N}$ ratio indicates if the $\mathrm{CN}$ cycle has been activated partially, or whether mixing and thermodynamic conditions have been such that the CN cycle has reached equilibrium. The latter is the case in hot-bottom burning (HBB), which is found in more massive AGB stars (Boothroyd et al. 1993). In this case, the bottom of the convective envelope connects with the H-burning shell, allowing processing of envelope $\mathrm{CN}$ material in the $\mathrm{H}$-shell. Material lost at the surface is accordingly modified in its $\mathrm{CN}$ abundance ratios. The limiting mass for the onset of $\mathrm{HBB}$ decreases with decreasing metallicity from $\sim 5 \mathrm{M}_{\odot}$ at $\mathrm{Z}=0.02$ to $\leq 3 \mathrm{M}_{\odot}$ for $\mathrm{Z}=0.0$ (Forestini \& Charbonnel 1997; Siess et al. 2002). Stars which experience $\mathrm{HBB}$ show a $\mathrm{C} / \mathrm{N}$ ratio close to the equilibrium ratio $(<0.1)$, as can be seen also in Figure 7 for the 4,5 , and $6 \mathrm{M}_{\odot}$ cases. On the contrary, the 2 and 3 $\mathrm{M}_{\odot}$ cases show very large $\mathrm{C} / \mathrm{N}$ ratios. Not even partial $\mathrm{CN}$ cycling has occurred in these models, and the small overabundance of $\mathrm{N}$ is entirely due to the first and second DUP (Herwig 2004). Partial CN cycling at the bottom of low-mass giant envelope convection is a well observed and parametrically modeled feature (e.g. Denissenkov \& VandenBerg 2003). Whether the same process operates in AGB stars as well is currently debated (e.g. Karakas et al. 2010).

\subsection{Considering the Effects of Dilution}

Nitrogen estimates only exist for ten of our stars. We compared the carbon and oxygen predictions from Herwig (2004) to all of our CEMP stars. None of the stars with available nitrogen estimates show the extremely low $\mathrm{C} / \mathrm{N}$ ratio indicative of hot-bottom burning. We therefore restrict the comparison of our sample to the 2 and 3 $\mathrm{M}_{\odot}$ models from Herwig (2004). In Figure 8, we show all of our stars that have $[\mathrm{C} / \mathrm{Fe}] \geq+1.0$. The black square and the red triangle at the upper right of the figure are the model predictions for $2 \mathrm{M}_{\odot}$ and $3 \mathrm{M}_{\odot}$, respectively. Clearly these predictions have higher carbon and oxygen estimates than our sample, but this is likely due to the fact that the effects of dilution are not considered. We consider a parametric mixing model to test the effects of dilution of the accreted material from an AGB companion. We chose a range of initial masses of the observed star $\left(0.5-0.9 \mathrm{M}_{\odot}\right)$ and accreted masses $\left(0.1-0.5 \mathrm{M}_{\odot}\right)$ and assumed complete mixing. We set the maximum mass of the observed star at $1 \mathrm{M}_{\odot}$. In order to consider only the AGB-phase contributions to carbon and oxygen, we subtracted off the likely contribution to carbon and oxygen arising from pre-star formation enhancements. These contributions were chosen to be the average enhancements of carbon and oxygen from the Spite et al. (2005) sample of unmixed metal-poor stars. The subtracted enhancement of $[\mathrm{C} / \mathrm{Fe}]$ was $0.18 \pm 0.16 \mathrm{dex}$ and the subtracted enhancement of $[\mathrm{O} / \mathrm{Fe}]$ was $0.7 \pm 0.17$ dex. The resulting predicted abundances based on this simple dilution experiment are shown as the black and the red lines in Figure 8. Once the effects of dilution are considered, the AGB predictions of Herwig (2004) fall within the parameter space of our sample. The magenta symbols are for the range of metallicity that is most consistent with the AGB models. For lower metallicities, the AGB model values for $[\mathrm{C} / \mathrm{Fe}]$ and $[\mathrm{O} / \mathrm{Fe}]$ would be larger, and for higher metallicity, they would decrease (Herwig 
2004).

\subsection{Uncertainties of the AGB Models}

The models of Herwig (2004) should be considered as rather conservative, standard predictions that suffer from the usual uncertainties associated mostly with convective mixing and mass loss. We can obtain an indication of the order of magnitude of these uncertainties by comparing the Herwig (2004) models with those of other authors. Comparing the $2 \mathrm{M}_{\odot}$ case with that of Cristallo et al. (2009a), the carbon predictions agree very well, while the $[\mathrm{N} / \mathrm{Fe}]$ prediction of Cristallo et al. (2009a) is about 0.2 dex higher and their O-overabundance prediction is 0.8 dex lower than that of Herwig (2004). Karakas (2010) provides a comparison between her $\mathrm{Z}=0.0001,2$ $\mathrm{M}_{\odot}$ yield predictions and that of Cristallo et al. (2009a) which shows that her $\mathrm{C}, \mathrm{N}$, and $\mathrm{O}$ yields are all approximately twice those of Cristallo et al. (2009a), which is easiest to be understood in terms of a lower mass loss rate in the Karakas (2010) yields. Karakas (2010) provides a more in-depth discussion of model prediction differences from different authors.

In addition, we have to discuss uncertainties deriving from entirely alternative evolution scenarios. Here, we should mention the possible occurrence of $\mathrm{H}$-ingestion flashes (HIF), which have been introduced in Section 6.2. These events are also referred to as proton-ingestion episodes or double He-shell flashes. There are several uncertainties related to these combustion-type flashes in which protons are convectively mixed into the He-shell flash convection zone, and release energy on the dynamic time scale of convective flows.

First, the occurrence of these events, in which the Heshell flash convection zone has to break through the entropy barrier associated with the $\mathrm{H}$-shell, is more likely with lower $\mathrm{CNO}$ abundances in the envelope. For example, an alpha-enhancement of the initial abundance composition without change of $[\mathrm{Fe} / \mathrm{H}]$ will make the HIF less likely or even suppress it (Cristallo et al. 2009a). If the stars are rotating (Meynet \& Maeder 2002), the CNO abundance may be enhanced during the core He-burning phase, which may also suppress the HIF (Herwig 2003).

The second uncertainty in HIF predictions is the quantitative mixing and nucleosynthesis in a convective combustion regime that breaks some of the assumptions of mixing-length theory and 1D spherically symmetric stellar evolution (Herwig et al. 2010). Keeping these significant uncertainties in mind, we nevertheless note that HIF models would predict the $\mathrm{N}$ signature of partial burning. For example, considering Figure 6 in Cristallo et al. (2009b), the prediction of a HIF model followed by maybe only a few pulses would correspond well to the CNO abundance patterns observed in most of our stars shown in Figure 7.

\section{CONCLUSIONS}

We have used near-IR medium-resolution spectroscopy in order to estimate $[\mathrm{O} / \mathrm{Fe}]$ for a sample of candidate carbon-enhanced stars selected from the Hamburg/ESO Survey. This method of abundance analysis allows us to obtain oxygen abundances accurate to about 0.4 dex. The use of four separate $\mathrm{CO}$ features to estimate oxygen abundances from the near-IR spectra allows for more pre- cise estimates, based on a robust average of the independently determined fits. A large spread of derived $[\mathrm{O} / \mathrm{Fe}]$ values are obtained for this sample, ranging from near the solar value to as much as one hundred times greater.

A comparison of our abundance determinations with high-resolution estimates was carried out. The values of $[\mathrm{O} / \mathrm{Fe}]$ for our full set of 57 CEMP stars largely fall within regions of parameter space occupied by the highresolution estimates of oxygen for other CEMP stars. We also found that the majority of our stars have oxygen abundances that are consistent with known CEMP-s and CEMP-r/s stars. Only a few stars could be considered CEMP-no stars, based on the data compiled in Masseron et al. (2010). This is likely due to the fact that CEMPno stars commonly have lower metallicities than most of the stars in this sample.

Oxygen enhancements (on the order of $[\mathrm{O} / \mathrm{Fe}]=+0.7$ ) have also been observed in very metal-poor stars without significant carbon enhancement, indicating that there were early oxygen-producing nucleosynthetic sites in the Galaxy independent of any enhancement by AGB evolution. However, we find that the $[\mathrm{C} / \mathrm{Fe}],[\mathrm{O} / \mathrm{Fe}]$, and $[\mathrm{N} / \mathrm{Fe}]$ (when available) estimates follow the patterns from Herwig (2004) closely enough that mass-transfer from a AGB companion is a likely scenario for many of the stars in our sample, especially when the effects of dilution are considered.

Our measured carbon abundances always exceed the available high-resolution $[\mathrm{N} / \mathrm{Fe}]$ abundances. If the origin of $\mathrm{CNO}$ abundance patterns comes from hot-bottomburning (HBB) in an intermediate mass (AGB) star, one would expect to see elevated $[\mathrm{N} / \mathrm{Fe}]$ relative to $[\mathrm{C} / \mathrm{Fe}]$ and $[\mathrm{O} / \mathrm{Fe}]$. This signature is not found in our sample, but it has been suggested that other mechanisms, such as cool-bottom-processing (Wasserburg et al. 1995; Denissenkov \& VandenBerg 2003) or the occurrence of HIF, can alter the levels of nitrogen enhancement.

It is likely that the majority of CEMP stars in this sample will turn out to be enhanced in neutron-capture elements. Consistency of most of our program stars with the CEMP-s class, based both on comparison to AGB models and existing high-resolution data, is expected since that CEMP stars with s-process-element enhancement are the most commonly observed type to date. However, recent chemical evolution models (Cescutti \& Chiappini 2010) have revealed that the winds from massive, rapidly-rotating metal-poor stars can result in a large scatter in the predicted abundances of $\mathrm{C}, \mathrm{N}$, and $\mathrm{O}$, presumably without the production of neutron-capture elements. Therefore, we are currently unable to assign classification to this sample of CEMP stars. High-resolution spectra of the stars in our sample will help clarify these questions.

C.R.K. and T.C.B. acknowledge partial support for this work from grants AST 07-07776, as well as from PHY 02-15783 and PHY 08-22648; Physics Frontier Center/Joint Institute or Nuclear Astrophysics (JINA), awarded by the US National Science Foundation. S.R. and V.M.P. thank MSU/JINA, FAPESP, CNPq and Capes for financial support. F.H. acknowledges funding through an NSERV Discovery Grant. 


\section{REFERENCES}

Alonso, A., Arribas, S., \& Martínez-Roger, C. 1996, A\&A, 313, 873

Aoki, W., Beers, T. C., Christlieb, N., Norris, J. E., Ryan, S. G., \& Tsangarides, S. 2007, ApJ, 655, 492

Beers, T. C., Preston, G. W., \& Schectman, S. A. 1985, AJ, 90, 2089

Beers, T. C., Preston, G. W., \& Shectman, S. A. 1992, AJ, 103, 1987

Beers, T. C., Rossi, S., Norris, J. E., Ryan, S. G., \& Shefler, T. 1999, AJ, 117, 981

Beers, T. C. \& Christlieb, N. 2005, ARA\&A, 43, 531

Beers, T. C., et al. 2007a, ApJ, 168, 128

Beers, T. C., Sivarani, T., Marsteller, B., Lee, Y. S., Rossi, S., \& Plez, B. 2007b, AJ, 133, 1193

Boothroyd, A. I., Sackmann, I. J., \& Ahern, S. C. 1993, ApJ, 416 762

Campbell, S. W. \& Lattanzio, J. C. 2008, A\&A, 490, 769

Cescutti, G., \& Chiappini, C. 2010, arXiv:1004.0088v1

Christlieb, N., Green, P.J., Wisotzki, L., \& Reimers, D. 2001, A\&A, 375, 366

Christlieb, N. 2003, RvMA, 16, 191

Christlieb, N., Schörck, T., Frebel, A., Beers, T. C., Wisotzki, L., \& Reimers, D. 2008, A\&A, 484, 721

Cohen, J. G. et al. 2005, ApJ, 633, L109

Collet, R., Asplund, M., \& Trampedach, R. 2006, ApJ, 644, L121

Collet, R., Asplund, M., \& Trampedach, R. 2007, A\&A, 469, 687

Cristallo, S., Straniero, O., Gallino, R., Piersanti, L., Domínguez, I., \& Lederer, M. T. 2009a, ApJ, 696, 797

Cristallo, S., Piersanti, L., Straniero, O., Gallino, R., Domínguez, I., \& Käppeler, F. 2009b, Publications of the ASA, 26, 139

Denissenkov, P. A., \& VandenBerg, D. A. 2003, ApJ, 593, 509

Denissenkov, P. A. \& Pinsonneault, M. 2008, ApJ, 679, 1541

Depoy, D. L., Atwood, B., Byard, P. L., Frogel, J., \& O'Brien, T. P. 1993, Society of Photo-Optical Instrumentation Engineers (SPIE) Conference Series, 1946, 667

Fabbian, D., Nissen, P. E., Asplund, M., Pettini, M., \& Akerman, C. 2009, A\&A, 500, 1143

Forestini, M. \& Charbonnel, C. 1997, A\&AS, 123, 241

Frebel, A. et al. 2005, Nature, 434, 871

Frebel, A. et al. 2006, ApJ, 652, 1585

Girardi, L., Bressan, A., Bertelli, G., \& Chiosi, C. 2000, A\&AS, 141,371

Goswami, A., Aoki, W., Beers, T. C., Christlieb, N., Norris, J. E., Ryan, S. G., \& Tsangarides, S. 2006, MNRAS, 372, 343

Goswami, A., Karinkuzhi, D., \& Shantikumar, N. S. 2010, MNRAS, 402, 1111

Gustafsson, B., Edvardsson, B., Eriksson, K., Jørgensen, U. G., Nordlund, A., \& Plez, B. 2008, A\&A, 486, 951

Hajduk, M. et al. 2005, Science, 308, 231

Herwig, F. 2003, ASP Conf. Ser., 304, 318

Herwig, F. 2004, ApJS, 155, 651

Herwig, F. 2005, ARA\&A, 43, 435

Herwig, F., Pignatari, M., Woodward, P. R., Porter, D. H., Rockefeller, G., Fryer, C. L., Bennett, M., \& Hirschi, R. 2010, arXiv: $1002.2241,1$

Hirschi, R., Fröhlich, C. Liebendörfer, M., \& Thielemann, F.-K. 2006, RvMA, 19, 101
Ito, H., Aoki, W., Honda, S., \& Beers, T.C. 2009, ApJ, 698, L37 Johnson, J. A., Herwig, F., Beers, T. C., Christlieb, N. 2007, ApJ, 658, 1203

Karakas, A. I. 2010, MNRAS, 403, 1413

Karakas, A. I., Campbell, S. W., \& Stancliffe, R. J. 2010, ApJ, 713,374

Kurucz, R. L. 1993, Kurucz CD-ROM 15, Diatomic Molecular Data for Opacity Calculations (Cambridge: SAO)

Lau, H. H. B., Stancliffe, R. J., \& Tout, C. A. 2009, MNRAS, 396,1046

Lucatello, S., Beers, T. C., Christlieb, N., Barklem, P. S., Rossi, S., Marsteller, B., Sivarani, T., Lee, Y. S. 2006, ApJ, 652, L37

Lugaro, M., Herwig, F., Lattanzio, J. C., Gallino, R., \& Straniero, O. 2003, ApJ, 586, 1305

Marigo, P, Girardi, L., Chiosi, C., \& Wood, P. R. 2001, A\&A, 371,152

Marsteller, B. E. "The Frequency of Carbon-Enhanced Metal-Poor Stars and the Origin of Carbon in the Universe", PhD diss., Michigan State University, 2007

Marsteller, B., Beers, T. C., Sivarani, T., Rossi, S., Placco, V. Knapp, G. R., Johnson, J. A., Lucatello, S. 2009, AJ, 138, 533

Masseron, T. et al. 2006, A\&A, 455, 1059

Masseron, T., Johnson, J. A., Plez, B., van Eck, S., Primas, F., Goriely, S., Jorissen, A. 2010, A\&A, 509, A93

Meléndez, J. \& Barbuy, B. 2002, ApJ, 575, 474

Meynet, G. \& Maeder, A. 2002, A\&A, 390, 561

Meynet, G., Ekström, S., \& Maeder, A. 2006, A\&A, 447, 623

Meynet, G., et al. 2010, in IAU Symposium 268, Light Elements in the Universe (ASP Conf. Series), eds. C. Charbonnel, M.

Tosi, F. Primas, and C. Chiappini, in press (arXiv:1001.1864)

Norris, J. E., Christlieb, N., Korn, A. J., Eriksson, K., Bessell, M. S., Beers, T. C., Wisotzki, L., Reimers, D. 2007, ApJ, 670, 774

Pettini, M., Ellison, S. L., Bergeron, J., \& Petitjean, P. 2002, A\&A, 391, 21

Pettini, M., Berkeley, J. Z., Steidel, C. C., Chaffee, F. H. 2008, MNRAS, 385, 2011

Placco, V. M. et al. 2010, AJ, 139, 1051

Plez, B. \& Cohen, J. G. 2005, A\&A, 434, 1117

Rossi, S., Beers, T. C., Sneden, C., Sevastyanenko, T., Rhee, J., Marsteller, B. 2005, 130, 2804

Schuler, S. C., Hatzes, A. P., King, J. R., Kürster, M., \& The, L. -S. 2006, AJ, 131, 1057

Siess, L., Livio, M., \& Lattanzio, J. 2002, ApJ, 570, 329

Sivarani, T., et al. 2006, A\&A, 459, 125

Skrutskie, M. F., et al. 2006, AJ, 131, 1163

Spite, M., et al. 2005, A\&A, 430, 655

Stancliffe, R. J., \& Glebbeek, E. 2008, MNRAS, 389, 1828

Tominaga, N., Umeda, H., \& Nomoto, K. 2007, ApJ, 660, 516

Tumlinson, J. 2006, AJ, 641, 1

Umeda, H., \& Nomoto, K. 2003, Nature, 422, 871

Umeda, H., \& Nomoto, K. 2005, ApJ, 619, 427

Wasserburg, G. J., Boothroyd, A. I., \& Sackmann, I.-J. 1995, AJ, 447, L37

Woodward, P., Herwig, F., Porter, D., Fuchs, T., Nowatzki, A., \& Pignatari, M. 2008, American Institute of Physics Conf. Ser., 990, First Stars III, 300 


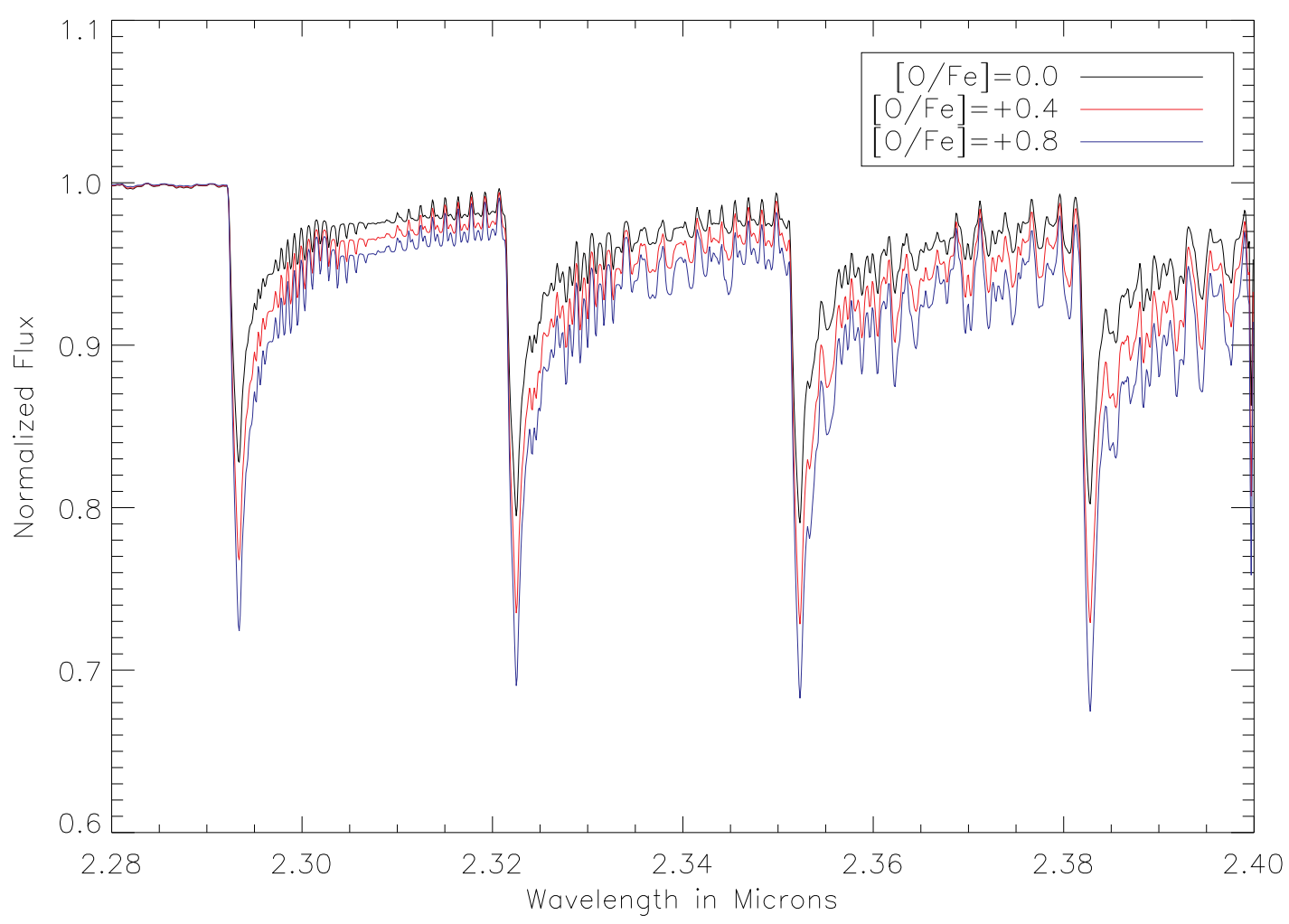

FIG. 1.- Three synthetic spectra with different $[\mathrm{O} / \mathrm{Fe}]$ ratios. Each spectrum has $T_{\text {eff }}$ of $4500 \mathrm{~K}, \log g$ of $1.0,[\mathrm{Fe} / \mathrm{H}]$ of -2.0 , and $[\mathrm{C} / \mathrm{Fe}]$ of +1.0 . 


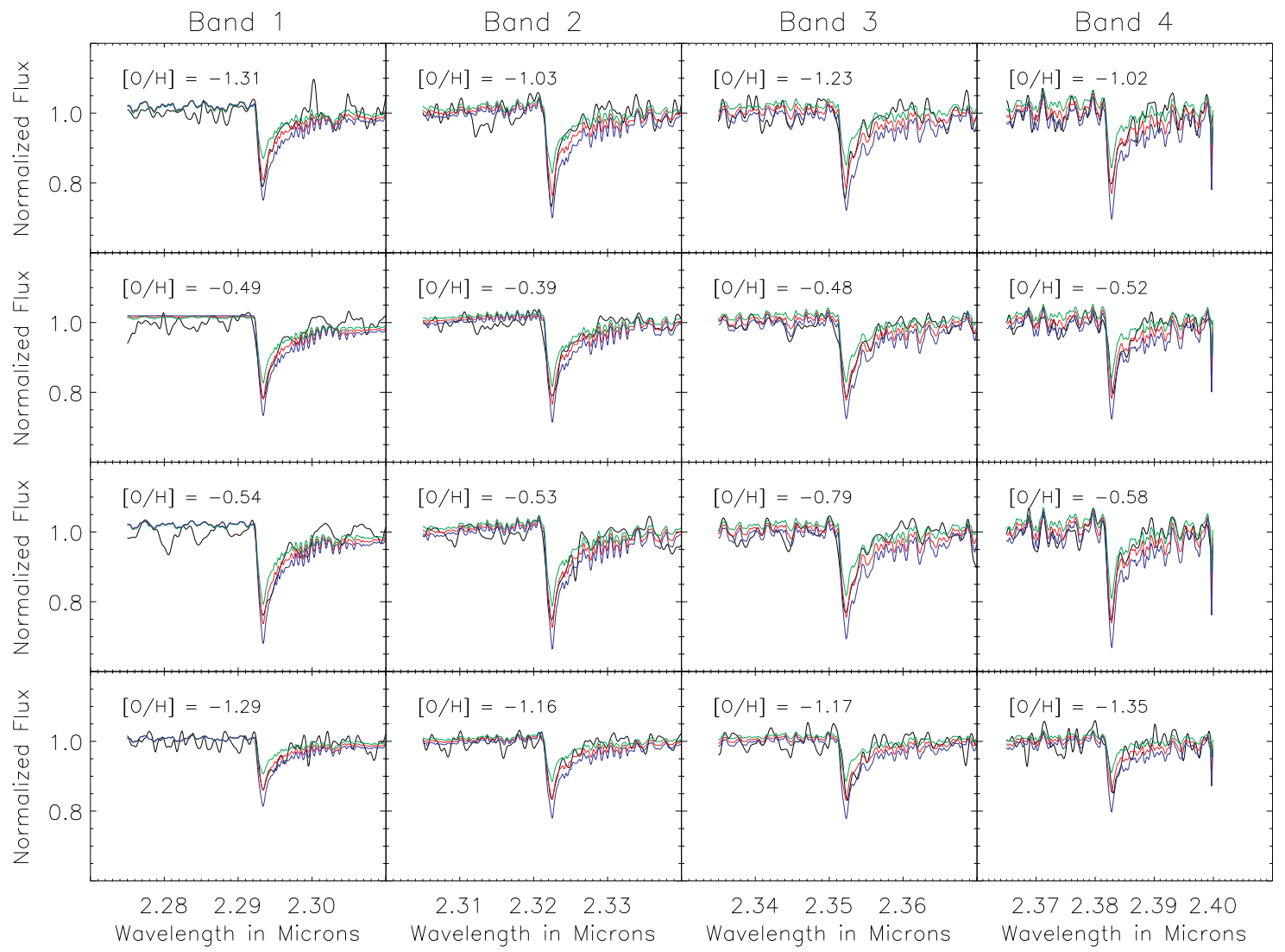

FIG. 2.- Each row shows four estimates of $[\mathrm{O} / \mathrm{H}]$ for a star from our sample: HE 0111-1346, HE 0519-2053, HE 1207-3156, and HE 2339-0837, respectively. In each panel, the black lines are the data, the red lines are the best-fitting synthetic spectra, the green lines have $[\mathrm{O} / \mathrm{H}]$ values of 0.5 dex lower than the best-fitting spectra, and the blue lines have $[\mathrm{O} / \mathrm{H}]$ values of 0.5 dex higher than the best-fitting spectra. A robust average of the four separate estimates is taken as the final estimate of oxygen abundance for each star. See text for details. 

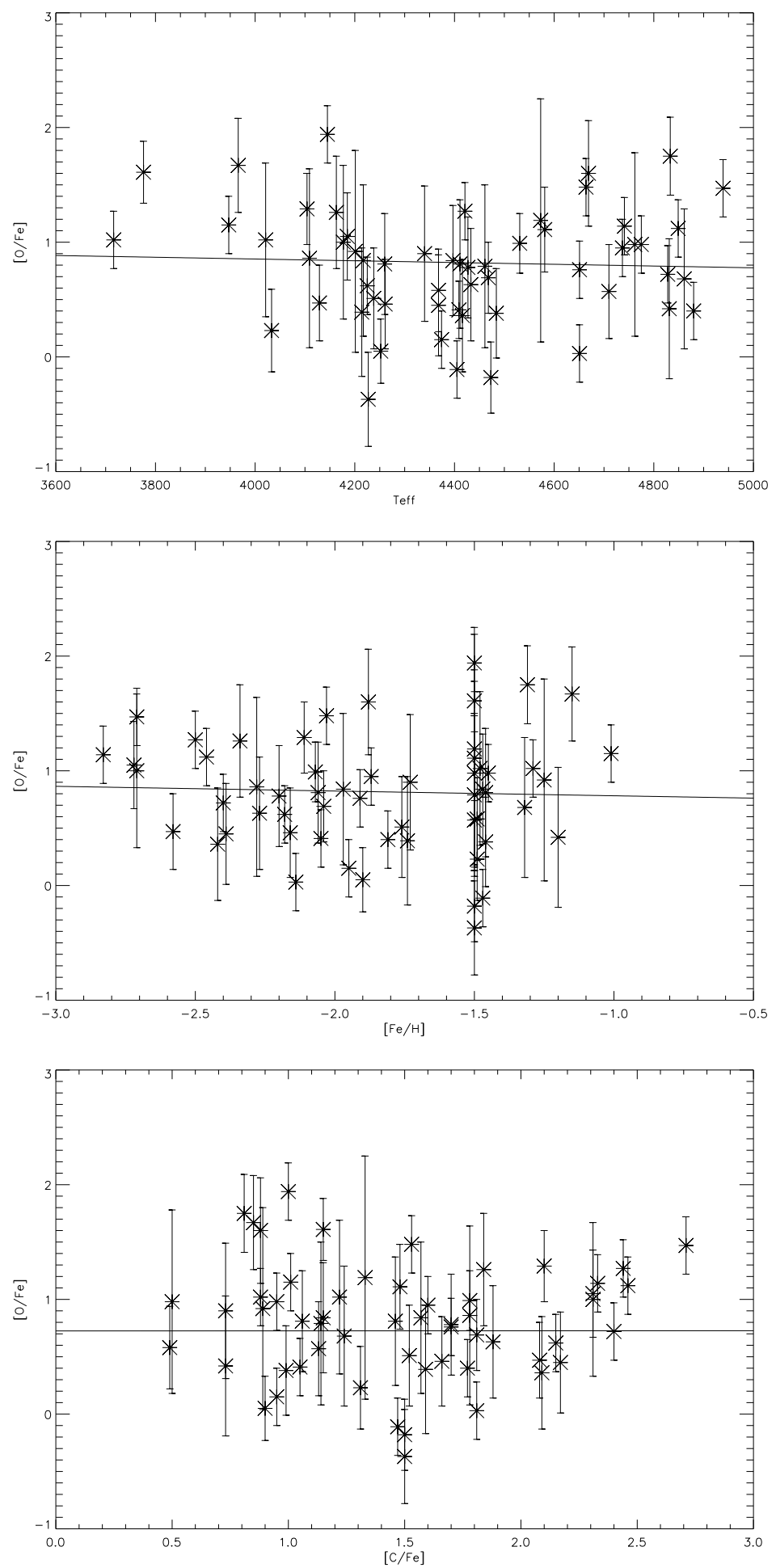

Fig. 3. - Top panel: $[\mathrm{O} / \mathrm{Fe}]$ vs. $T_{\text {eff }}$ for the entire sample. Middle panel: $[\mathrm{O} / \mathrm{Fe}]$ vs. $[\mathrm{Fe} / \mathrm{H}]$ for the entire sample. Bottom panel: $[\mathrm{O} / \mathrm{Fe}]$ vs. $[\mathrm{C} / \mathrm{Fe}]$ for the entire sample. 


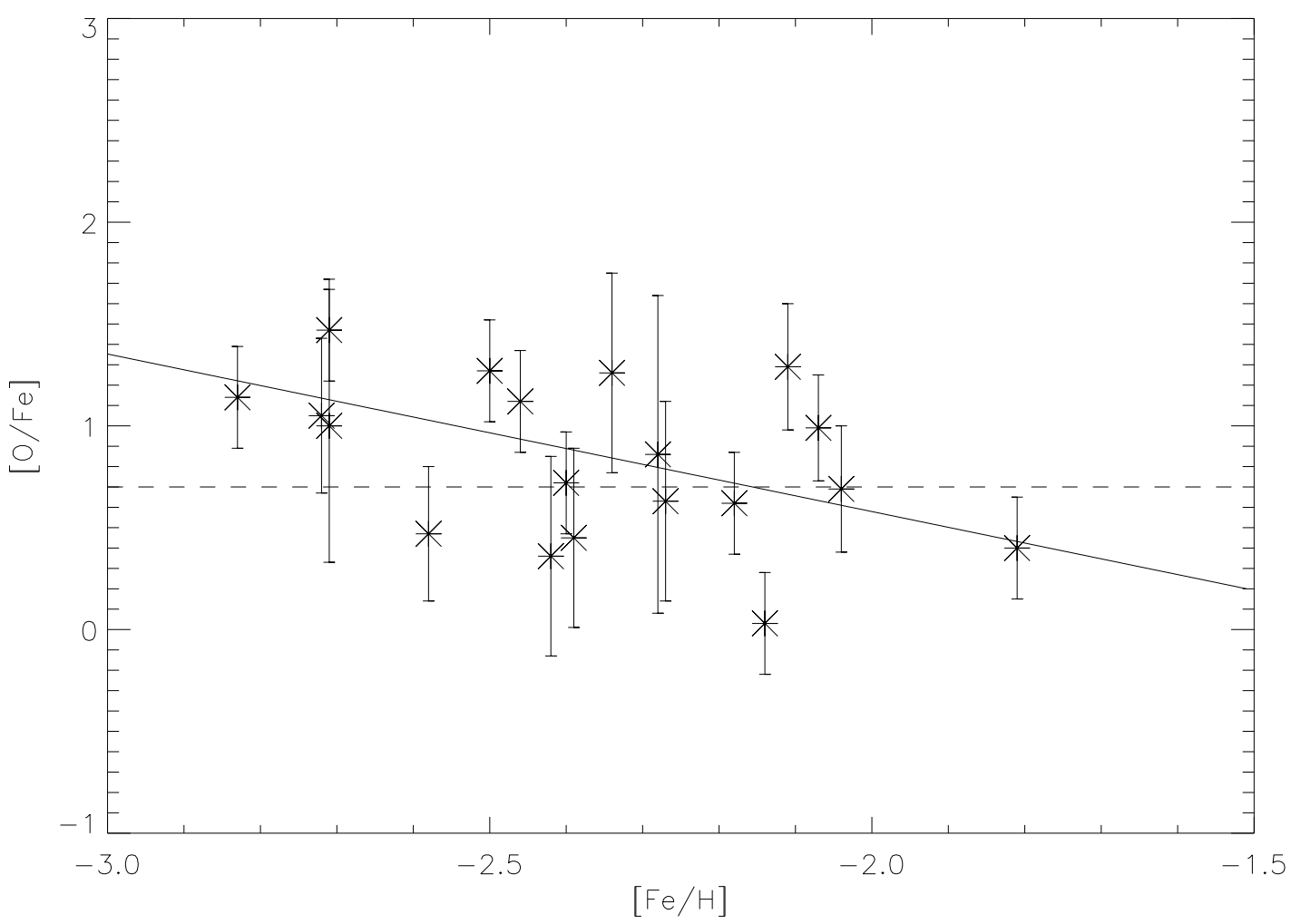

Fig. 4. $-[\mathrm{O} / \mathrm{Fe}]$ vs. $[\mathrm{Fe} / \mathrm{H}]$ for the stars with $[\mathrm{C} / \mathrm{Fe}] \geq+1.75$. The dashed line represents the fit for the carbon-normal stars from the Spite et al. (2005) sample of very metal-poor stars, while the solid line is the best fit for our data. 


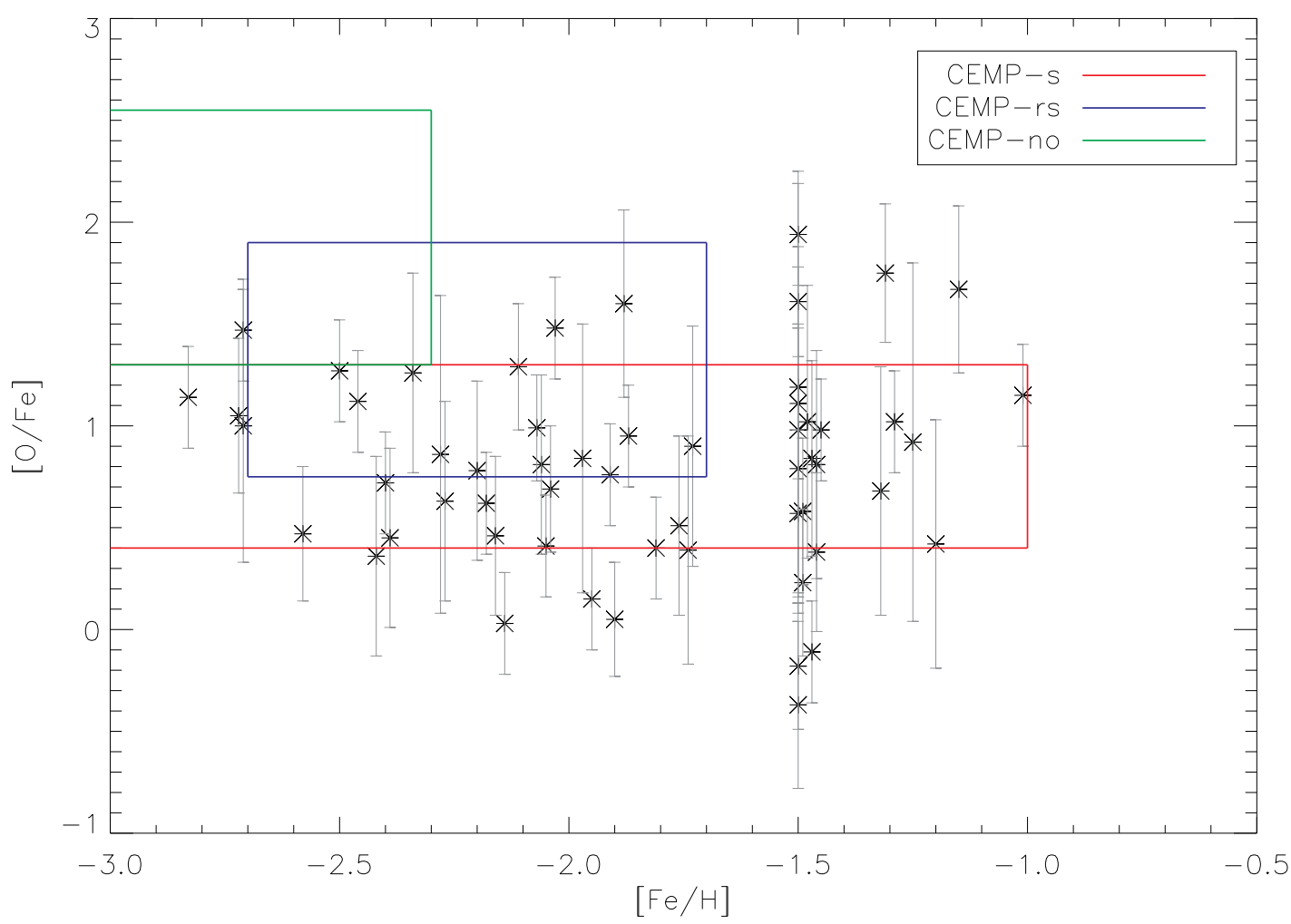

FIG. 5.- $[\mathrm{O} / \mathrm{Fe}]$ vs. $[\mathrm{Fe} / \mathrm{H}]$ for the entire sample. The colored boxes show the regions occupied by different types of CEMP stars found in Masseron et al. (2010). 


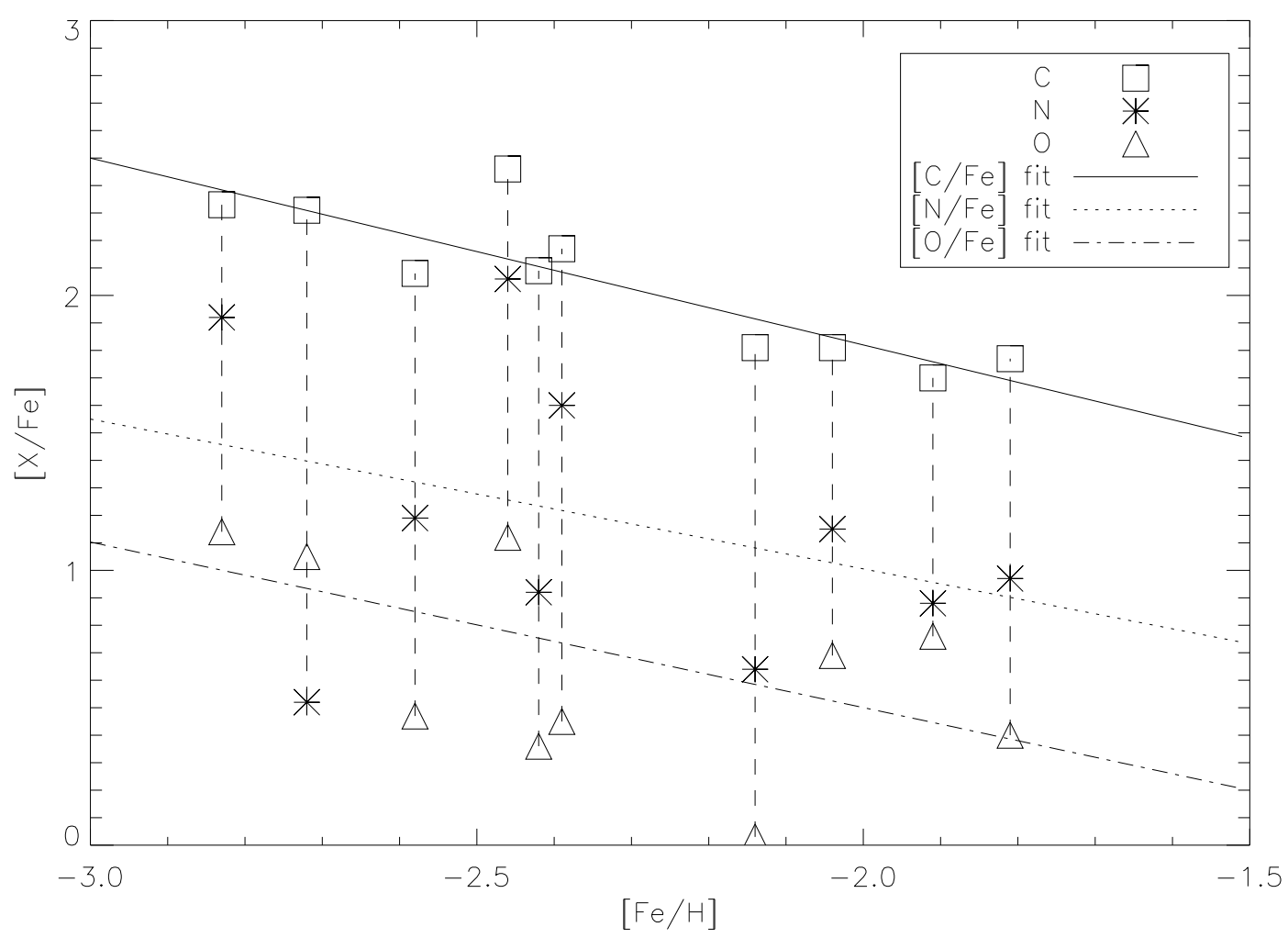

FIG. 6.- C, N, and O abundances vs. metallicity for 10 stars from our sample. Also shown are linear fits for these species. 


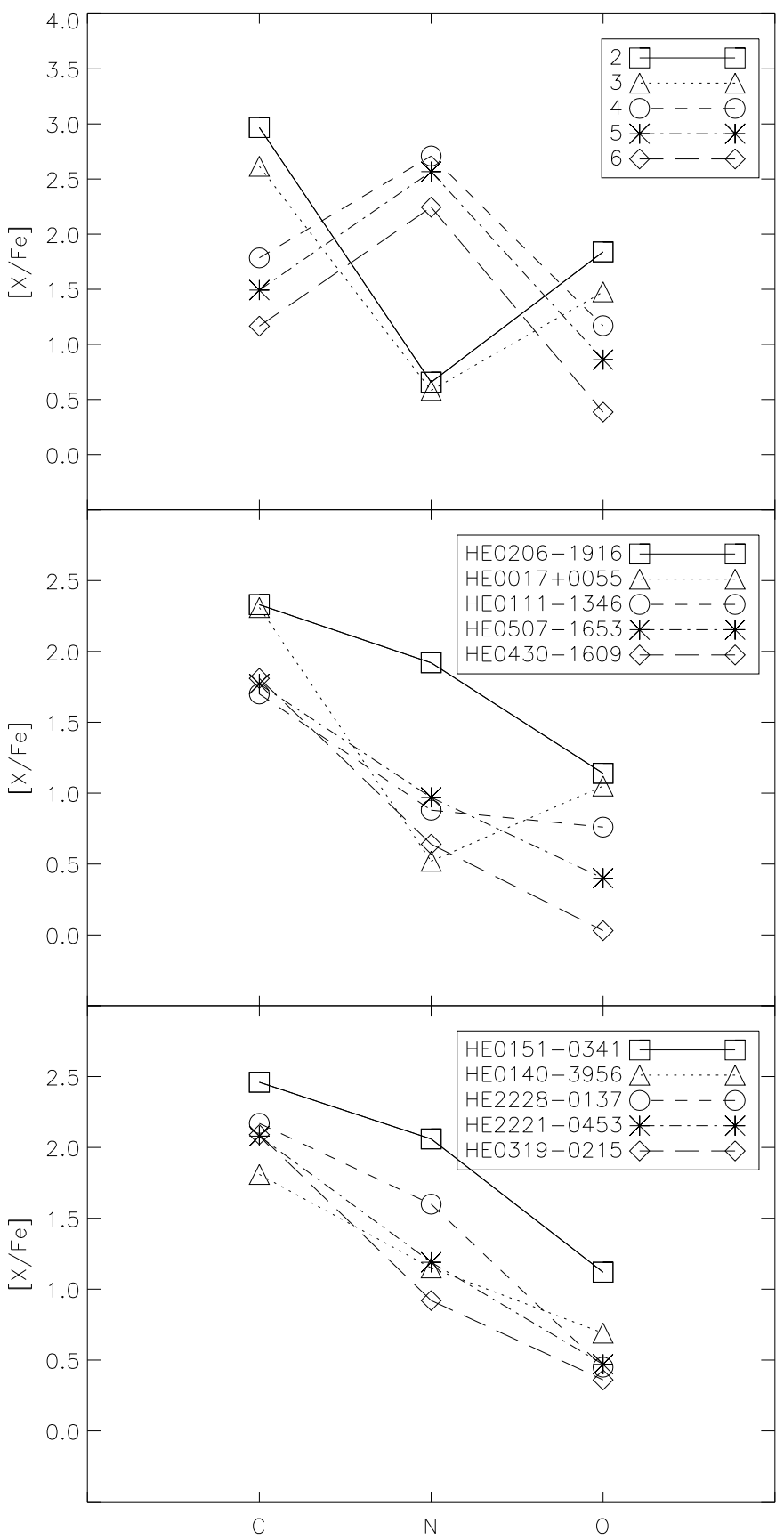

FIG. 7.- Top panel: Predicted abundances of C, N, and O for model AGB stars of different masses (in M $\odot$ ) from Herwig (2004). Middle and bottom panels: Abundances of $\mathrm{C}, \mathrm{N}$, and $\mathrm{O}$ for 10 stars from our sample. 


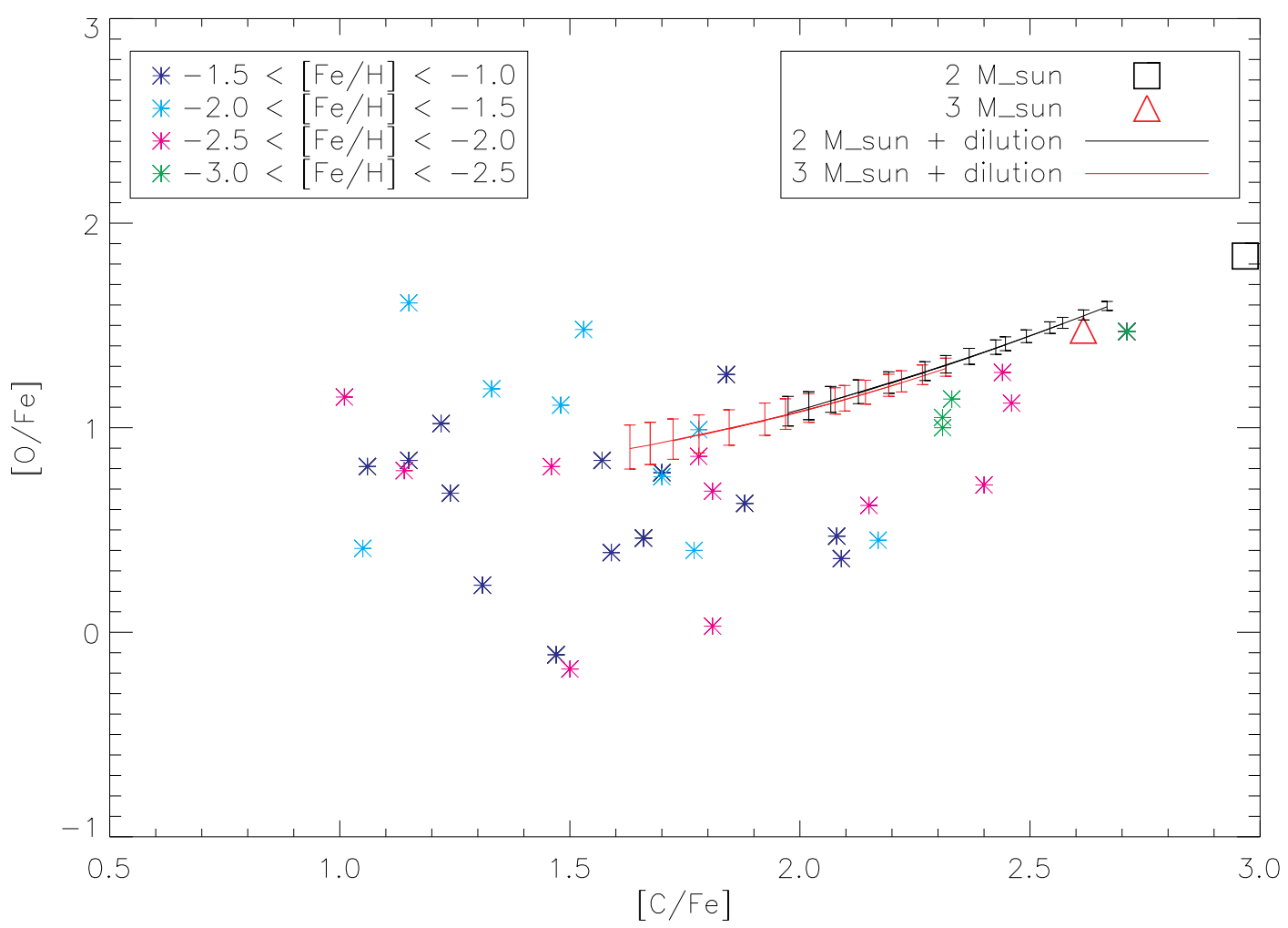

FIG. 8. $-[\mathrm{O} / \mathrm{Fe}]$ vs. $[\mathrm{C} / \mathrm{Fe}]$ for all stars from our sample with $[\mathrm{C} / \mathrm{Fe}] \geq+1.0$, color-coded by their metallicity ranges, as noted in the legend. The black square and red triangle mark the values of the abundances predicted by Herwig (2004) and the colored lines represent the effects of dilution once the AGB material is accreted onto the observed CEMP star. See text for more details. 
TABLE 1

Estimates of Atmospheric Parameters and C, N, O Abundance Ratios

\begin{tabular}{|c|c|c|c|c|c|c|c|c|c|c|}
\hline Name & $T_{\text {eff }}(\mathrm{K})$ & $\log g(\operatorname{cgs})$ & {$[\mathrm{Fe} / \mathrm{H}]$} & {$[\mathrm{C} / \mathrm{H}]$} & {$[\mathrm{C} / \mathrm{Fe}]$} & {$[\mathrm{C} / \mathrm{Fe}]_{h}$} & {$[\mathrm{~N} / \mathrm{Fe}]_{h}$} & {$[\mathrm{O} / \mathrm{H}]$} & {$[\mathrm{O} / \mathrm{Fe}]$} & $\sigma_{[\mathrm{O} / \mathrm{Fe}]}$ \\
\hline HE $0002+0053$ & 4225 & 0.27 & -2.18 & -0.03 & 2.15 & $\cdots$ & $\ldots$ & -1.6 & 0.6 & 0.3 \\
\hline HE $0010-3051$ & 4177 & 0.17 & -2.71 & -0.40 & 2.31 & $\ldots$ & $\ldots$ & -1.7 & 1.0 & 0.7 \\
\hline HE $0017+0055$ & 4185 & 0.18 & -2.72 & -0.41 & 2.31 & 1.82 & 0.52 & -1.7 & 1.0 & 0.4 \\
\hline HE $0033-5605$ & 4021 & 0.00 & -1.48 & -0.26 & 1.22 & $\ldots$ & $\ldots$ & -0.5 & 1.0 & 0.7 \\
\hline HE $0043-2433$ & 4397 & 0.61 & -1.47 & -0.32 & 1.15 & $\ldots$ & $\ldots$ & -0.6 & 0.8 & 0.5 \\
\hline HE $0111-1346$ & 4651 & 1.08 & -1.91 & -0.22 & 1.70 & 1.48 & 0.88 & -1.2 & 0.8 & 0.3 \\
\hline HE $0120-5834$ & 4828 & 1.62 & -2.40 & 0.00 & 2.40 & $1.79^{\mathrm{a}}$ & $1.59^{\mathrm{a}}$ & -1.7 & 0.7 & 0.3 \\
\hline HE $0140-3956$ & 4468 & 0.84 & -2.04 & -0.23 & 1.81 & 1.55 & 1.15 & -1.4 & 0.7 & 0.3 \\
\hline HE $0151-0341$ & 4849 & 1.42 & -2.46 & 0.00 & 2.46 & 2.16 & 2.06 & -1.3 & 1.1 & 0.3 \\
\hline HE $0155-2221$ & 4109 & 0.00 & -2.28 & -0.50 & 1.78 & $\ldots$ & $\ldots$ & -1.4 & 0.9 & 0.8 \\
\hline HE $0206-1916$ & 4741 & 1.23 & -2.83 & -0.50 & 2.33 & 2.42 & 1.92 & -1.7 & 1.1 & 0.3 \\
\hline HE 0219-1739 & 4227 & 0.27 & -1.50 & 0.00 & 1.50 & $0.31^{\mathrm{a}}$ & $0.31^{\mathrm{a}}$ & -1.9 & -0.4 & 0.4 \\
\hline HE $0251-2118$ & 4710 & 1.16 & -1.50 & -0.37 & 1.13 & $\ldots$ & $\ldots$ & -0.9 & 0.6 & 0.4 \\
\hline HE $0310+0059$ & 4861 & 1.69 & -1.32 & -0.07 & 1.24 & $\ldots$ & $\ldots$ & -0.6 & 0.7 & 0.6 \\
\hline HE $0314-0143$ & 4201 & 0.22 & -1.25 & -0.36 & 0.89 & $\ldots$ & $\ldots$ & -0.3 & 0.9 & 0.9 \\
\hline HE $0319-0215$ & 4416 & 0.64 & -2.42 & -0.33 & 2.09 & 2.12 & 0.92 & -2.1 & 0.4 & 0.5 \\
\hline HE $0330-2815$ & 4411 & 0.64 & -1.46 & 0.00 & 1.46 & $\ldots$ & $\ldots$ & -0.7 & 0.8 & 0.6 \\
\hline HE $0359-0141$ & 4340 & 0.54 & -1.73 & -1.00 & 0.73 & $\ldots$ & $\ldots$ & -0.8 & 0.9 & 0.6 \\
\hline HE $0408-1733$ & 4260 & 0.33 & -2.06 & -1.00 & 1.06 & $\ldots$ & $\ldots$ & -1.3 & 0.8 & 0.4 \\
\hline HE $0417-0513$ & 4669 & 1.22 & -1.88 & -1.00 & 0.88 & $\ldots$ & $\ldots$ & -0.3 & 1.6 & 0.5 \\
\hline HE $0419+0124$ & 4368 & 0.61 & -1.49 & -1.00 & 0.49 & $\ldots$ & $\ldots$ & -0.9 & 0.6 & 0.4 \\
\hline HE $0429+0232$ & 4409 & 0.63 & -2.05 & -1.00 & 1.05 & $\ldots$ & $\ldots$ & -1.6 & 0.4 & 0.3 \\
\hline HE $0430-1609$ & 4651 & 1.08 & -2.14 & -0.33 & 1.81 & 1.84 & 0.64 & -2.1 & 0.0 & 0.3 \\
\hline HE $0439-1139$ & 4833 & 1.62 & -1.31 & -0.50 & 0.81 & $\ldots$ & $\ldots$ & 0.4 & 1.8 & 0.3 \\
\hline HE $0457-1805$ & 4484 & 0.77 & -1.46 & -0.46 & 0.99 & $\ldots$ & $\ldots$ & -1.1 & 0.4 & 0.4 \\
\hline HE $0458-1754$ & 4374 & 0.56 & -1.95 & -1.00 & 0.95 & $\ldots$ & $\cdots$ & -1.8 & 0.2 & 0.3 \\
\hline HE $0507-1653$ & 4880 & 1.50 & -1.81 & -0.04 & 1.77 & 1.61 & 0.97 & -1.4 & 0.4 & 0.3 \\
\hline HE $0518-1751$ & 4252 & 0.32 & -1.90 & -1.00 & 0.90 & $\ldots$ & $\ldots$ & -1.9 & 0.1 & 0.3 \\
\hline HE $0519-2053$ & 4775 & 1.46 & -1.45 & -0.50 & 0.95 & $\ldots$ & $\ldots$ & -0.5 & 1.0 & 0.3 \\
\hline HE $0547-4428$ & 4217 & 0.25 & -1.97 & -0.40 & 1.57 & $\ldots$ & $\ldots$ & -1.1 & 0.9 & 0.7 \\
\hline HE 1011-0942 & 3716 & 5.00 & -1.29 & -0.41 & 0.88 & $\ldots$ & $\ldots$ & -0.3 & 1.0 & 0.3 \\
\hline HE $1023-1504$ & 4421 & 0.66 & -2.50 & -0.06 & 2.44 & $\ldots$ & $\ldots$ & -1.2 & 1.3 & 0.3 \\
\hline HE $1125-2942$ & 3947 & 5.00 & -1.01 & 0.00 & 1.01 & $\cdots$ & $\cdots$ & 0.1 & 1.2 & 0.3 \\
\hline HE $1145-0002$ & 4033 & 0.00 & -1.49 & -0.18 & 1.31 & $\ldots$ & $\ldots$ & -1.3 & 0.2 & 0.4 \\
\hline HE $1152-0355$ & 4214 & 0.23 & -1.74 & -0.15 & 1.59 & $\ldots$ & $\ldots$ & -1.4 & 0.4 & 0.6 \\
\hline HE $1152-0430$ & 4573 & 0.93 & -1.50 & -0.17 & 1.33 & $\ldots$ & $\ldots$ & -0.3 & 1.2 & 1.1 \\
\hline HE $1204-0600$ & 4581 & 1.07 & -1.50 & -0.02 & 1.48 & $\ldots$ & $\ldots$ & -0.4 & 1.1 & 0.4 \\
\hline HE $1207-3156$ & 4664 & 1.23 & -2.03 & -0.50 & 1.53 & $\ldots$ & $\ldots$ & -0.6 & 1.5 & 0.3 \\
\hline HE $1230-0230$ & 3966 & 5.00 & -1.15 & -0.30 & 0.85 & $\cdots$ & $\cdots$ & 0.5 & 1.7 & 0.4 \\
\hline HE $1238-0435$ & 4433 & 0.68 & -2.27 & -0.39 & 1.88 & $\ldots$ & $\ldots$ & -1.6 & 0.6 & 0.5 \\
\hline HE $1246-1510$ & 3776 & 5.00 & -1.50 & -0.35 & 1.15 & $\ldots$ & $\ldots$ & 0.1 & 1.6 & 0.3 \\
\hline HE $1255-2324$ & 4405 & 0.69 & -1.47 & 0.00 & 1.47 & $\cdots$ & $\cdots$ & -1.6 & -0.1 & 0.3 \\
\hline HE $1331-0247$ & 4238 & 0.28 & -1.76 & -0.25 & 1.52 & $\ldots$ & $\ldots$ & -1.3 & 0.5 & 0.4 \\
\hline HE $1410-0125$ & 4427 & 0.66 & -2.20 & -0.50 & 1.70 & $\ldots$ & $\ldots$ & -1.4 & 0.8 & 0.4 \\
\hline HE $1418+0150$ & 4163 & 0.13 & -2.34 & -0.50 & 1.84 & $\cdots$ & $\ldots$ & -1.1 & 1.3 & 0.5 \\
\hline HE $1428-1950$ & 4531 & 0.85 & -2.07 & -0.28 & 1.78 & $\ldots$ & $\ldots$ & -1.1 & 1.0 & 0.3 \\
\hline HE $1431-0755$ & 4104 & 0.00 & -2.11 & -0.01 & 2.10 & $\ldots$ & $\ldots$ & -0.8 & 1.3 & 0.3 \\
\hline HE $1524-0210$ & 4145 & 5.00 & -1.50 & -0.50 & 1.00 & $\ldots$ & $\ldots$ & 0.4 & 1.9 & 0.3 \\
\hline HE $2115-0522$ & 4762 & 1.46 & -1.50 & -1.00 & 0.50 & $\ldots$ & $\ldots$ & -0.5 & 1.0 & 0.8 \\
\hline HE $2145-1715$ & 4461 & 0.77 & -1.50 & -0.36 & 1.14 & $\ldots$ & $\ldots$ & -0.7 & 0.8 & 0.7 \\
\hline HE $2153-2323$ & 4261 & 0.33 & -2.16 & -0.50 & 1.66 & $1.13^{\mathrm{a}}$ & $0.93^{\mathrm{a}}$ & -1.7 & 0.5 & 0.4 \\
\hline HE $2200-1652$ & 4473 & 0.84 & -1.50 & 0.00 & 1.50 & $\ldots$ & $\cdots$ & -1.7 & -0.2 & 0.3 \\
\hline HE $2207-1746$ & 4737 & 1.23 & -1.87 & -0.27 & 1.60 & $\ldots$ & $\ldots$ & -0.9 & 1.0 & 0.3 \\
\hline HE $2221-0453$ & 4129 & 0.04 & -2.58 & -0.50 & 2.08 & 2.08 & 1.19 & -2.1 & 0.5 & 0.3 \\
\hline HE $2224-0330$ & 4831 & 1.62 & -1.20 & -0.47 & 0.73 & $\ldots$ & $\ldots$ & -0.8 & 0.4 & 0.6 \\
\hline HE $2228-0137$ & 4368 & 0.61 & -2.39 & -0.22 & 2.17 & 1.90 & 1.60 & -1.9 & 0.5 & 0.4 \\
\hline HE $2339-0837$ & 4939 & 1.60 & -2.71 & 0.00 & 2.71 & $\ldots$ & $\ldots$ & -1.2 & 1.5 & 0.3 \\
\hline
\end{tabular}

a These values are not used for analysis due to a large discrepancy in estimated $[\mathrm{Fe} / \mathrm{H}]$. 